\title{
Use of a stop valve to enhance disinfectant exposure may improve sink drain disinfection
}

\author{
Jennifer L. Cadnum BS ${ }^{1}$, Scott H. Livingston $\mathrm{BS}^{1,2}$, Scott A. Gestrich $\mathrm{MD}^{1}$, Annette L. Jencson BS, $\mathrm{CIC}^{1}$, \\ Brigid M. Wilson $\mathrm{PhD}^{3}$ and Curtis J. Donskey $\mathrm{MD}^{2,3}$ \\ ${ }^{1}$ Research Service, Louis Stokes Cleveland VA Medical Center, Cleveland, Ohio, ${ }^{2}$ Case Western Reserve University School of Medicine Cleveland, Ohio and \\ ${ }^{3}$ Geriatric Research, Education, and Clinical Center, Louis Stokes Cleveland VA Medical Center, Cleveland, Ohio
}

To the Editor-Sinks in healthcare facilities are a potential reservoir for dissemination of multidrug-resistant gram-negative bacilli and Candida spp. ${ }^{1,2}$ Unfortunately, sink drainage systems provide a favorable environment for pathogen colonization and biofilm formation, but they are not amenable to cleaning and disinfection. ${ }^{3}$ Pouring disinfectants into sink drains has been reported to be beneficial, but reductions in sink colonization have often been limited or transient. ${ }^{4}$ We hypothesized that disinfectants poured into drains might have limited efficacy in part because they flow rapidly down the drain, providing inadequate contact time and poor penetration into many of the areas harboring microorganisms. Thus, we conducted a pilot study to test the hypothesis that approaches that allow instillation of disinfectant throughout the proximal drainage system for a prolonged period would improve the efficacy of liquid disinfectants.

In initial experiments, we installed a stop valve immediately distal to the $\mathrm{P}$ trap in a research laboratory sink known to be colonized with Pseudomonas aeruginosa. Closure of the valve resulted in stoppage of flow; opening the valve allowed liquids to flow normally. We tested the impact of 3 interventions: (1) pouring $500 \mathrm{~mL}$ of $5 \%$ acetic acid down the drain over 1 minute; (2) pouring $500 \mathrm{~mL}$ of a 1 to 10 dilution of household bleach down the drain over 1 minute; and (3) pouring $500 \mathrm{~mL}$ of a 1 to 10 dilution of household bleach down the drain over 1 minute with the stop valve closed allowing complete filling of the drainage system from the valve to just above the strainer for 1 hour followed by opening of the valve and flushing with water for 30 seconds. To assess the impact of the interventions, quantitative cultures for gram-negative bacilli were collected from the proximal sink drain to depth of $2.5 \mathrm{~cm}$ below the strainer before treatment and intermittently for 15 days post treatment. The experiment was repeated twice for each intervention.

A second set of experiments was conducted in 4 patient rooms with sink colonization by gram-negative bacilli. Among the 4 sinks, 2 were treated by pouring $500 \mathrm{~mL}$ of disinfectant down the drain and the other 2 were treated by instillation of the disinfectant for 1 hour. The experimental methods were as described previously with the following exceptions: (1) the valve was placed proximal to the $\mathrm{P}$ trap approximately $15 \mathrm{~cm}$ below the strainer; (2) the disinfectant

Author for correspondence: Curtis J. Donskey MD, Geriatric Research, Education, and Clinical Center 1110W, Louis Stokes Cleveland VA Medical Center, 10701 East Boulevard, Cleveland, Ohio 44106. E-mail: Curtis.Donskey@va.gov

Cite this article: Cadnum JL, et al. (2019). Use of a stop valve to enhance disinfectant exposure may improve sink drain disinfection. Infection Control \& Hospital Epidemiology 2019, 40, 254-256. doi: 10.1017/ice.2018.318 used was a commercial improved hydrogen peroxide product; and (3) the sinks were operated by patients and staff as needed. The experiment was repeated twice; sinks treated with one method during the first run were treated with the opposite method during the second run. For both sets of experiments, an ordinary least-squares regression model was used to compare treatment groups.

As shown in Fig. 1(A), pouring bleach or acetic acid into the laboratory sink resulted in only transient suppression of proximal drain colonization, whereas instillation of bleach for 1 hour resulted in suppression at this site for several days $(P<.001)$. The organisms recovered throughout the experiment were identified as $P$. aeruginosa.

As shown in Fig. 1(B), pouring the improved hydrogen peroxide disinfectant into the patient room sinks resulted in only transient suppression of proximal drain colonization, whereas instillation proximal to the $\mathrm{P}$ trap for 1 hour reduced gramnegative bacilli concentrations in the section below the strainer for several days $(P<.001)$. After 1 hour of disinfectant instillation, numerous visible pieces of organic and inorganic material were released from the sides of the drain pipes, and swabs used to sample the sink drain were visibly cleaner than baseline swabs (Fig. 1(C)).

Our results demonstrate that pouring disinfectants down drains has only a transient impact on the microbial load just below the strainer. In contrast, use of a valve to allow instillation of disinfectant throughout the proximal drainage system with a 1-hour dwell time resulted in reduced proximal sink drain colonization for several days. This effect was probably observed due to increased disinfectant contact time and enhanced penetration into some of the areas harboring microorganisms. These findings are clinically relevant because the proximal sink drain is the primary site of dispersal from colonized sinks. ${ }^{5}$ Moreover, the fact that colonization of the drain was reduced for several days suggests that intermittent rather than daily application of the disinfection process might be effective in reducing the risk for dispersal.

Our study has some limitations. The study was a small proofof-concept study that will require validation in larger studies. However, some previous studies have suggested that similar approaches can be effective in reducing sink contamination. ${ }^{6,7}$ For example, Klick et $\mathrm{al}^{6}$ reported that daily filling of sinks fitted with drain cutoff valves with $5 \%$ phenol solution in conjunction with heating was effective in reducing colonization with Pseudomonas spp. We did not demonstrate that the reduction in proximal sink drain colonization resulted in reduced dispersal of organisms during operation of the sinks. Finally, installing valves in sink 
A



B

- Improved hydrogen peroxide instillation $\square$ Improved hydrogen peroxide poured

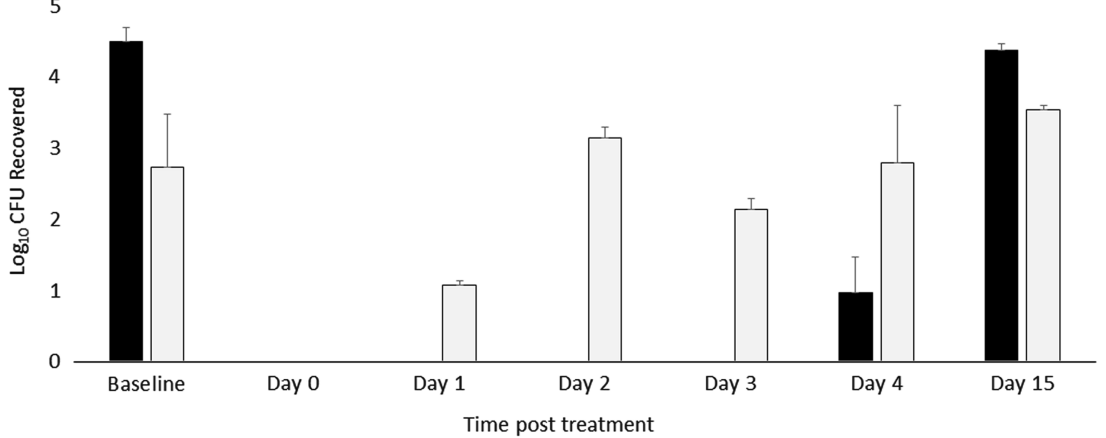

C

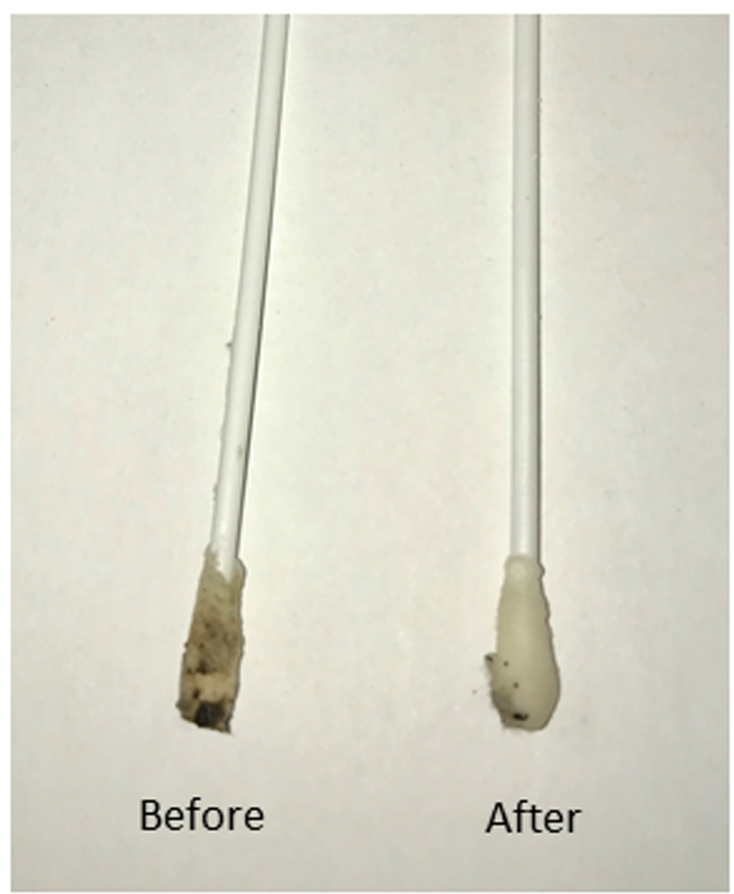

Fig. 1. Efficacy of pouring versus instilling $500 \mathrm{~mL}$ of disinfectants on recovery of gram-negative bacilli from proximal sink drains in a research laboratory sink (A) and in sinks in rooms of hospitalized patients $(\mathrm{N}=4)(\mathrm{B})$. For instillation, a stop valve was used to allow complete filling of the drainage system from the valve to just above the strainer for 1 hour. Quantitative cultures for gram-negative bacilli were collected from the proximal sink drain to depth of $2.5 \mathrm{~cm}$ below the strainer before treatment and intermittently for 15 days post treatment. For the laboratory sink, the stop valve was just distal to the $\mathrm{P}$ trap, whereas for the patient room sinks it was just proximal to the $\mathrm{P}$ trap. Swabs collected before versus after instillation of disinfectant for 1 hour are shown in panel (C). 
drainage systems may not be feasible for some sink designs and could be costly and labor intensive. Thus, we are currently investigating several simpler approaches to achieve the same effect.

Acknowledgments. We would like to thank the staff of the Cleveland VA Medical Center's Engineering Service for assistance in designing and installing sink valves.

Financial support. This work was supported by the Department of Veterans Affairs.

Conflicts of interest. C.J.D. has received research funding from Clorox, GOJO, Pfizer, Avery Dennison, PDI, and Boehringer Laboratories. All other authors report no potential conflicts.

\section{References}

1. Kizny Gordon AE, Mathers AJ, Cheong EYL, et al. The hospital water environment as a reservoir for carbapenem-resistant organisms causing hospital-acquired infections-a systematic review of the literature. Clin Infect Dis 2017 May 15;64:1435-1444.
2. Jencson AL, Cadnum JL, Piedrahita C, Donskey CJ. Hospital sinks are a potential nosocomial source of Candida infections. Clin Infect Dis 2017;65:1954-1955.

3. Livingston SH, Cadnum JL, Gestrich S, Jencson AL, Donskey CJ. A novel sink drain cover prevents dispersal of microorganisms from contaminated sink drains. Infect Control Hosp Epidemiol 2018;39: 1254-1256.

4. Parkes LO, Hota SS. Sink-related outbreaks and mitigation strategies in healthcare facilities. Curr Infect Dis Rep 2018;20:42.

5. Kotay S, Chai W, Guilford W, Barry K, Mathers AJ. Spread from the sink to the patient: in situ study using green fluorescent protein (GFP)expressing Escherichia coli to model bacterial dispersion from hand washing sink trap reservoirs. Appl Environ Microbiol 2017;83(8):pii: e03327-16.

6. Klick JM, du Moulin GC, Hedley-Whyte J, Teres D, Bushnell LS, Feingold DS. Prevention of gram-negative bacillary pneumonia using polymyxin aerosol as prophylaxis. II. Effect on the incidence of pneumonia in seriously ill patients. J Clin Invest 1975;55:514-519.

7. Gbaguidi-Haore H, Varin A, Cholley P, Thouverez M, Hocquet D, Bertrand X. A bundle of measures to control an outbreak of Pseudomonas aeruginosa associated with P-trap contamination. Infect Control Hosp Epidemiol 2018;39:164-169.

\title{
A novel color additive for bleach wipes indicates surface coverage and contact time to improve thoroughness of cleaning
}

\author{
Kevin Tyan BA, Katherine Jin BA and Jason Kang BSc \\ Kinnos, Brooklyn, New York
}

To the Editor-Healthcare-associated infections (HAIs) exact a heavy toll on the US healthcare system, affecting $\sim 1.7$ million patients and resulting in direct costs of up to $\$ 45$ billion each year. ${ }^{1}$ Institutions are heavily emphasizing frequent disinfection of hightouch surfaces to prevent transmission to patients because contaminated surfaces are known to be reservoirs for nosocomial pathogens. ${ }^{2}$ In particular, hospitals have increasingly adopted the approach of daily cleaning with ready-to-use bleach wipes to combat $C$. difficile infections (CDIs). Daily cleaning with a sporicidal agent was demonstrated to be the most effective single intervention against CDI and asymptomatic colonization, ${ }^{3}$ while one hospital reported a reduction of $85 \%$ in $\mathrm{CDI}$ following the implementation of daily bleach wipe cleaning in all patient rooms. ${ }^{4}$

However, effective disinfection requires proper technique, adequate training, and constant monitoring. ${ }^{5}$ Significant human error in disinfectant wiping practices has been well documented, with personnel missing high-touch surfaces, overusing single wipes, and inadvertently transferring pathogens between surfaces, or drying off the applied disinfectant before the necessary wet-contact time. ${ }^{5}$ Periodic assessments of cleaning compliance through adenosine triphosphate (ATP) bioluminescence assays and fluorescent marker systems may help mitigate human error, but improvements cannot be sustained without permanent systematic changes and constant

Author for correspondence: Kevin Tyan, 760 Parkside Avenue, Suite 215, Brooklyn, NY 11226. E-mail: kevin@kinnos.us

Cite this article: Tyan K, et al. (2019). A novel color additive for bleach wipes indicates surface coverage and contact time to improve thoroughness of cleaning. Infection Control \& Hospital Epidemiology 2019, 40, 256-258. doi: 10.1017/ice.2018.323 feedback. This assertion is evidenced by a report in which environmental services (EVS) staff improved their cleaning performance from $52 \%$ to $83 \%$ after implementing fluorescent marker monitoring but regressed toward the baseline (57\%) after monthly feedback ceased. ${ }^{6}$ Current methods for quality control monitoring are retrospective and often are only intermittently performed; thus, it may be challenging to translate to timely feedback for EVS staff. To sustain a high level of cleaning compliance, a unique approach is needed, one that provides direct and immediate feedback to workers.

A novel attachment to bleach wipe containers, the Highlight Wipes Lid (Kinnos, Brooklyn, NY) administers a blue indicator onto dispensed bleach wipes to provide real-time visual feedback of the thoroughness of surface coverage and the passage of contact time. ${ }^{7}$ As shown in Fig. 1A, the device consists of (1) a reusable lid that attaches onto standard containers of commercially available bleach wipes, and (2) a disposable cartridge containing the Highlight blue liquid additive and pre-installed batteries. A user presses the button to dispense individual wipes imbued with the blue indicator through the front face of the battery-powered lid. This dispensing mechanism was designed for ease of use and prevention of bleach splash-back that typically occurs when manually pulling wipes through standard lid orifices. Furthermore, the lid automatically retracts hanging wipes back into the canister after a period of inactivity to prevent drying of the bleach wipe, loss of efficacy, and wastage of the productproblems common in currently used bleach wipes.

Figure $1 \mathrm{~B}$ compares the visibility of surface coverage using bleach wipes alone and bleach wipes dispensed through the Highlight Wipes Lid. When wiped on a standard bedside rail 